\title{
Parasite-Probiotic Interactions in the Gut: Bacillus sp. and Enterococcus faecium Regulate Type-2 Inflammatory Responses and Modify the Gut Microbiota of Pigs During Helminth Infection
}

Myhill, Laura J.; Stolzenbach, Sophie; Mejer, Helena; Krych, Lukasz; Jakobsen, Simon R.; Kot, Witold; Skovgaard, Kerstin; Canibe, Nuria; Nejsum, Peter; Nielsen, Dennis S.

Total number of authors:

12

Published in:

Frontiers in Immunology

Link to article, DOI:

10.3389/fimmu.2021.793260

Publication date:

2022

Document Version

Publisher's PDF, also known as Version of record

Link back to DTU Orbit

Citation (APA):

Myhill, L. J., Stolzenbach, S., Mejer, H., Krych, L., Jakobsen, S. R., Kot, W., Skovgaard, K., Canibe, N., Nejsum, P., Nielsen, D. S., Thamsborg, S. M., \& Williams, A. R. (2022). Parasite-Probiotic Interactions in the Gut: Bacillus $\mathrm{sp}$. and Enterococcus faecium Regulate Type-2 Inflammatory Responses and Modify the Gut Microbiota of Pigs During Helminth Infection. Frontiers in Immunology, 12, [793260]. https://doi.org/10.3389/fimmu.2021.793260

\section{General rights}

Copyright and moral rights for the publications made accessible in the public portal are retained by the authors and/or other copyright owners and it is a condition of accessing publications that users recognise and abide by the legal requirements associated with these rights.

- Users may download and print one copy of any publication from the public portal for the purpose of private study or research.

- You may not further distribute the material or use it for any profit-making activity or commercial gain

- You may freely distribute the URL identifying the publication in the public portal 


\section{OPEN ACCESS}

Edited by:

Silvia Melgar

University College Cork, Ireland

Reviewed by:

Friederike Ebner,

Free University of Berlin, Germany

Anastasia N. Vlasova,

The Ohio State University,

United States

*Correspondence:

Andrew R. Williams

arw@sund.ku.dk

${ }^{\dagger}$ These authors have contributed equally to this work

Specialty section: This article was submitted to

Mucosal Immunity,

a section of the journal

Frontiers in Immunology

Received: 11 October 2021

Accepted: 07 December 2021

Published: 05 January 2022

Citation:

Myhill LJ, Stolzenbach S, Mejer H, Krych L, Jakobsen SR, Kot W,

Skovgaard K, Canibe N, Nejsum P,

Nielsen DS, Thamsborg SM and

Williams AR (2022) Parasite-Probiotic

Interactions in the Gut: Bacillus sp. and

Enterococcus faecium Regulate

Type-2 Inflammatory Responses

and Modify the Gut Microbiota of

Pigs During Helminth Infection.

Front. Immunol. 12:793260.

doi: 10.3389/fimmu.2021.793260
Parasite-Probiotic Interactions in the Gut: Bacillus sp. and Enterococcus faecium Regulate Type-2 Inflammatory Responses and Modify the Gut Microbiota of Pigs During Helminth Infection

\author{
Laura J. Myhill ${ }^{1 \dagger}$, Sophie Stolzenbach ${ }^{1 \dagger}$, Helena Mejer ${ }^{1}$, Lukasz Krych ${ }^{2}$, \\ Simon R. Jakobsen ${ }^{1}$, Witold Kot ${ }^{3}$, Kerstin Skovgaard ${ }^{4}$, Nuria Canibe ${ }^{5}$, Peter Nejsum ${ }^{6}$, \\ Dennis S. Nielsen ${ }^{2}$, Stig M. Thamsborg ${ }^{1 \dagger}$ and Andrew R. Williams ${ }^{1 *+}$

\begin{abstract}
${ }^{1}$ Department of Veterinary and Animal Sciences, University of Copenhagen, Faculty of Health and Medical Sciences, Frederiksberg, Denmark, ${ }^{2}$ Department of Food Science, University of Copenhagen, Frederiksberg, Denmark, ${ }^{3}$ Department of Plant and Environmental Sciences, University of Copenhagen, Frederiksberg, Denmark, ${ }^{4}$ Department of Biotechnology and Biomedicine, Technical University of Denmark, Kongens Lyngby, Denmark, ${ }^{5}$ Department of Animal Science - Immunology
\end{abstract} \\ and Microbiology, Aarhus University, Tjele, Denmark, ${ }^{6}$ Department of Clinical Medicine, Aarhus University, Aarhus, Denmark
}

Dietary probiotics may enhance gut health by directly competing with pathogenic agents and through immunostimulatory effects. These properties are recognized in the context of bacterial and viral pathogens, but less is known about interactions with eukaryotic pathogens such as parasitic worms (helminths). In this study we investigated whether two probiotic mixtures (comprised of Bacillus amyloliquefaciens, B. subtilis, and Enterococcus faecium [BBE], or Lactobacillus rhamnosus LGG and Bifidobacterium animalis subspecies Lactis Bb12 [LB]) could modulate helminth infection kinetics as well as the gut microbiome and intestinal immune responses in pigs infected with the nodular worm Oesophagostomum dentatum. We observed that neither probiotic mixture influenced helminth infection levels. BBE, and to a lesser extent LB, changed the alphaand beta-diversity indices of the colon and fecal microbiota, notably including an enrichment of fecal Bifidobacterium spp. by BBE. However, these effects were muted by concurrent $O$. dentatum infection. BBE (but not LB) significantly attenuated the O. dentatum-induced upregulation of genes involved in type-2 inflammation and restored normal lymphocyte ratios in the ileo-caecal lymph nodes that were altered by infection. Moreover, inflammatory cytokine release from blood mononuclear cells and intestinal lymphocytes was diminished by BBE. Collectively, our data suggest that selected probiotic mixtures can play a role in maintaining immune homeostasis during type 2-biased inflammation. In addition, potentially beneficial changes in the microbiome induced by dietary probiotics may be counteracted by helminths, highlighting the complex inter-relationships that potentially exist between probiotic bacteria and intestinal parasites.

Keywords: probiotics, swine, helminths, Oesophagostomum dentatum, type-2 immune response, gut microbiota 


\section{INTRODUCTION}

The mammalian gut environment is maintained in a complex homeostasis encompassing interactions between dietary compounds, the commensal gut microbiota (GM) and the mucosal immune system (1). Dysregulation of this balanced ecosystem can lead to increased susceptibility to pathogen infection and chronic inflammation, and is a major source of disease and morbidity in humans and decreased productivity in livestock. To this end, dietary supplementation with probiotic bacteria has gained increasing attention as a safe method to maintain intestinal homeostasis, subsequently improving gut health. Beneficial effects of probiotics are strain-specific and dose-dependent, and can be achieved by modulating intestinal motility and barrier function, outcompeting enteropathogens, or by modifying the composition of host GM, subsequently affecting host mucosal immune responses $(2,3)$.

Pigs are a key species in the food production industry and also serve as an important model for human biomedical research due to similarities in gastrointestinal physiology and microbiota composition (4). Supplementation of pig diets with probiotics has revealed beneficial effects such as improved growth, carcass quality, and enhanced host protective responses against different pathogens, with pronounced efficiency at reducing bacterial load of enterotoxigenic Escherichia coli (F4) in weaned piglets (5-9). Additional studies against eukaryotic pathogens have also reported beneficial effects of probiotics. For example, in vitro and murine models of Giardia infection have shown that Lactobacillus spp. and Enterococcus faecium can eliminate infection and reinforce host immune responses (10-12).

Parasitic worms (helminths) are among the most widespread gut pathogens, infecting more than a billion humans worldwide and being commonly found in nearly all farmed livestock $(13,14)$. Infection can result in marked immunopathology and a reduction in mucosal barrier function and poses a significant risk to health and productivity $(15,16)$. Moreover, mucosal-dwelling helminths induce strongly polarized Thelper (Th) type- 2 immunity and thus serve as a useful model for Th2-mediated intestinal immune responses, such as those elicited by food allergens (17). Studies on the trilateral interactions between parasites, the GM and the immune system may therefore shed light on the role of gut bacteria in regulating host-parasite and immune interactions at mucosal barrier surfaces (1). Several studies have reported that feeding prebiotic dietary fibres (e.g. inulin) or administration of microbial metabolites (short-chain fatty acids (SCFA) or lactic acid) can strongly influence infection dynamics and immune responses induced by the large intestinal-dwelling parasites Trichuris suis (porcine whipworm) and Oesophagostomum dentatum (porcine nodular worm) (18-20). These effects are thought to be mediated by GM changes in the caecum and colon $(20,21)$, as inulin is known to increase the abundance of microbes such as Lactobacillaceae and Bifidobacterium during helminth infection (20).

Reports on the effects of dietary probiotic supplementation during helminth infection are limited, and whether probiotics can modulate helminth infection and associated inflammatory and immunopathological changes in the large intestine, as appears to be the case with inulin and other prebiotics, remains unknown. Supplementation with Bifidobacterium animalis subspecies Lactis Bb12 was shown to modulate mucosal immune responses and enhance jejunal barrier function in pigs infected with Ascaris suum (22), whilst Lactobacillus rhamnosus LGG intake supressed the development of type-2 related immune responses in the trachealbronchial lymph nodes of A. suum-infected pigs (23). Thus, probiotic bacteria may exert immunomodulatory effects in the context of type- 2 immune function. In light of this, porcine models of helminth infection may represent a valuable model for studying the interactions between probiotic bacteria and gut pathogens, and assessing if probiotics have potential as healthpromoting dietary additives that can prevent or alleviate the effects of enteric helminth infection.

$O$. dentatum infections are common in pig production systems worldwide. Infection is accompanied by a type-2biased inflammatory response in the mucosal tissue at the site of infection (the caecum and proximal colon), however this is often insufficient to clear the parasites and chronic infections may develop over several months, before a slow immunemediated expulsion begins (24). O. dentatum appears to be sensitive to changes in the gut environment, as direct infusion of SCFA or lactic acid can result in worm expulsion (19). Thus, this infection model serves as an economically and biologically relevant system for testing the effects of probiotic bacteria on the outcome of a helminth infection. Here, we investigated the effects of two different probiotic mixtures on O. dentatum establishment and infection dynamics. In addition, we explored the interactions between these probiotics and infection on GM composition throughout the intestinal tract, as well as peripheral and local mucosal immune responses. We show that a dynamic relationship exists between probiotic supplementation, the GM and the immune system during helminth infection, which may have significant implications for our understanding of the regulation of type-2 inflammation in mucosal tissues, and for the application of probiotics for prevention or control of intestinal diseases.

\section{MATERIALS AND METHODS}

\section{Experimental Design}

A total of 48 Yorkshire-Landrace pigs (females and castrated males, 8-10 weeks old, initial body weight approximately $20 \mathrm{~kg}$ ) were sourced from a specific pathogen-free farm. After stratification based on sex and weight, pigs were randomly allocated to one of six groups. Each treatment group contained eight pigs housed in a separate pen. Two groups (each $n=8$ ) received the basal diet only (based on ground barley and soybean containing 16.2\% crude protein). Two groups (each $n=8$ ) received the same diet supplemented with BBE containing the strains Bacillus amyloliquefaciens 516 (porcine origin), B. subtilis 541 (human origin), and Enterococcus faecium 669 (human origin). The final two groups (each $n=8$ ) received the basal diet supplemented with $\mathrm{LB}$, containing the strains Lactobacillus rhamnosus $\mathrm{LGG}^{\circledR}$ (human origin; DSM33156) and Bifidobacterium animalis subsp. Lactis BB$12^{\circledR}$ (food origin; DSM15954). All probiotic strains were supplied by 
Chr.Hansen A/S, Denmark. Pigs were fed twice a day with the probiotic-supplements mixed with the standard feed immediately before feeding. For both probiotic mixtures, pigs received $2 \times 10^{10}$ CFU per day.

After two weeks of diet adaptation, a total of 24 pigs (8 pigs from each diet treatment group) were each inoculated with $25 \mathrm{O}$. dentatum third stage larvae (L3)/kg body weight, by oral gavage. These pigs subsequently continued to receive the same $O$. dentatum L3 dose three days a week until study end (a total of four weeks). Infection doses were provided during the morning feeding, and were uniformly distributed on top of the feed. The dosed feed was provided in troughs that allowed all pigs' adequate space to feed equally and simultaneously. The dosing regime was chosen to mimic a natural moderate exposure level and the average approximate theoretical total dose during the study was 22,000 O. dentatum L3/pig. The remaining 24 pigs were uninfected for the duration of the study.

All pigs had been vaccinated against $L$. intercellularis with one dose of a live, attenuated vaccine (Enterisol ${ }^{\circledR}$ Ileitis, Boehringer Ingelheim) on farm four weeks prior to arriving on the experimental premises (which was six weeks prior to infection with $O$. dentatum). All pigs were confirmed negative for $O$. dentatum infection upon arrival by McMaster fecal egg count and serology. For the duration of the study, all pigs were housed on concrete floored pens with wood chips and water provided ad libitum. Welfare checks were performed daily, with body weight monitored and reported weekly. At day 28 post-infection (p.i.), 48 pigs were sacrificed over the course of three days by stunning with captive bolt followed by exsanguination.

\section{Digesta Sampling and O. dentatum Isolation}

Weekly blood and fecal samples were taken between arrival (day -14) and until the end of the study (day 28 p.i.). Blood samples were taken in order to collect serum for ELISA (see below), and isolate peripheral blood mononuclear cells (PBMCs; day 28 p.i. only). Faecal samples were scored following a 5-point scale (1 hard; 2 - normal; 3 - soft; 4 - watery; 5 - diarrhoea) in order to monitor changes in fecal consistency as a result of probiotic supplementation. After scoring, samples were cooled to $\sim 4^{\circ} \mathrm{C}$ immediately upon collection for subsequent enumeration of $O$. dentatum egg counts per gram of faeces (EPG) using a McMaster fecal egg count method (as described in Roepstorff \& Nansen, 1998) (25).

At necropsy, fresh intestinal digesta samples were collected from specific intestinal sections: jejunum (mid-point of the small intestine), ileum (10 cm proximal from ileocecal junction), caecum, proximal colon (20 cm distal from ileocecal junction) and distal colon (central part of the spiral) colon) for microbiota and $\mathrm{pH}$ measurement, with additional samples taken from the proximal colon for SCFA analysis, as previously described (26). Following this, $O$. dentatum larvae and adults were recovered according to the agar-gel migration technique described previously by Slotved et al. (27). Briefly, luminal contents of caecum and colon were collected and diluted to a total volume of 10 litres using $0.9 \%$ saline $\left(37^{\circ} \mathrm{C}\right)$. A $5 \%$ sub-sample was then embedded in $2 \%$ agar on cloths that were then suspended in saline and incubated for 24 hours at $37^{\circ} \mathrm{C}$ to isolate immature and adult $O$. dentatum from each pig. Worms were isolated on a $38 \mu \mathrm{m}$ mesh and stored in $70 \%$ ethanol for later enumeration. For each pig, ten adult female and male worms were selected for length measurement, using Leica Application Suite version 4.7 (Leica Microsystems, Germany), as a measure of $O$. dentatum fitness.

\section{Cell Isolation, Flow Cytometry, and Assessment of Cytokine Production}

Ileo-caecal lymph nodes (CLNs) were dissected and passed through a $70 \mu \mathrm{M}$ cell strainer to obtain single cell suspensions. After a series of washing, the cells were prepared for analysis of frequencies of $\mathrm{T}$ cell, $\mathrm{B}$ cell and monocyte populations as described in Myhill et al. (20). Flow cytometry was performed using a BD Accuri C6 flow cytometer (BD Biosciences), and data were analysed using Accuri CFlow Plus software (Accuri ${ }^{\circledR}$ Cytometers Inc., MI, USA). PBMCs were isolated from heparinised whole blood using Histopaque-1077 (SigmaAldrich) and centrifugation. To assess cytokine production, isolated CLN cells were cultured for 48 hours in complete media (RPMI 1640 supplemented with $2 \mathrm{mM}$ L-glutamine, $10 \%$ calf serum, $100 \mu \mathrm{g} / \mathrm{mL}$ streptomycin and $100 \mathrm{U} / \mathrm{mL}$ penicillin) together with $10 \mu \mathrm{g} / \mathrm{mL}$ phytohemagglutinin (Sigma-Aldrich). Measurement of secreted TNF $\alpha$ and IL-10 was assessed using commercial ELISA kits (R\&D systems). Isolated PBMCs in complete media were stimulated with LPS $(1 \mu \mathrm{g} / \mathrm{mL})$, cultured for 24 hours, and concentrations of IL-6, TNF $\alpha$, IL-10 and IL-1 $\beta$ assessed by ELISA. Values below the detection limit were assigned an arbitrary value of half the lowest value of the standard curve.

\section{O. dentatum Culture}

O. dentatum larvae were isolated from infected control-fed pigs, and washed extensively in $37^{\circ} \mathrm{C}$ saline. The exsheathed larvae were cultured in complete media containing antibiotics and fungicide for 3 days at $37^{\circ} \mathrm{C}$ to obtain excretory/secretory (E/S) products. Every day the culture media was removed, stored at $-80^{\circ} \mathrm{C}$, and replaced with fresh media. Pooled culture media containing E/S was concentrated by centrifugation using Amicon ultra centrifugal filter units (MWCO $10 \mathrm{kDa}$, Sigma-Aldrich, Denmark), and filtered prior to testing of protein content by bicinchoninic (BCA) assay (Thermo Fisher Scientific).

\section{O. dentatum ELISA}

Anti-O. dentatum IgA and IgG 1 levels in serum were quantified by ELISA as described in Myhill et al. (20). Briefly, plates (Nunc Maxisorb) were coated with $5 \mu \mathrm{g} / \mathrm{mL}$ O. dentatum larval E/S overnight at $4^{\circ} \mathrm{C}$. Serum antibodies were then detected using goat anti-pig IgA-horseradish peroxidase (HRP; BioRad, Germany), or mouse anti-pig $\operatorname{IgG}_{1}$ (clone K139-3C8; BioRad) followed by goat anti-mouse IgG-HRP conjugate (BioRad). Incubations were for 1 hour at $37^{\circ} \mathrm{C}$, and between all steps, plates were washed four times with PBS plus $0.02 \%$ Tween 20 . After development with tetramethylbenzidine (TMB) substrate, the reaction was stopped 
with $0.2 \mathrm{M} \mathrm{H}_{2} \mathrm{SO}_{4}$, and the plates read at $450 \mathrm{nM}$ with a Multiskan FC plate reader (Waltham, Massachusetts, USA).

\section{Quantitative Real-Time PCR}

Total RNA was extracted from proximal colon tissue using a miRNAeasy ${ }^{\circledR}$ Mini kit (Qiagen, CA, USA) according to manufacturer's guidelines, and as described in Myhill et al. (20). Synthesis of cDNA and pre-amplification was conducted as described in Williams et al. (28). A panel of 77 genes of interest, including key Th1/Th2/Treg/innate immune response-related genes and epithelial/mucosal barrier function-related genes, were examined on a BioMark HD Reader (Fluidigm). First, a thermal mix and hot start protocol was performed to mix primers, samples and reagents $\left(50^{\circ} \mathrm{C}\right.$ for $2 \mathrm{~min}, 70^{\circ} \mathrm{C}$ for $30 \mathrm{~min}, 25^{\circ} \mathrm{C}$ for $10 \mathrm{~min}$, $50^{\circ} \mathrm{C}$ for 2 minutes, $95^{\circ} \mathrm{C}$ for $10 \mathrm{~min}$ ), followed by qPCR using the following cycling conditions of: 35 cycles at $95^{\circ} \mathrm{C}$ for 15 seconds and $60^{\circ} \mathrm{C}$ for $1 \mathrm{~min}$. After data pre-processing, 68 genes of interest passed quality control criteria and were statistically analysed. Three pigs (two in the infected, control-fed group and one in the uninfected, BBE-fed group) were excluded due to not passing quality controls (insufficient cDNA amplification) Normalization using several validated reference housekeeping genes and data preprocessing, was carried out as described in Skovgaard et al. (29). Primer sequences are presented in Supplementary Table 1. Raw relative expression data is available in Supplementary Data Sheet 1.

\section{S rRNA Sequencing of Microbiota}

DNA was extracted from faeces or intestinal content in a randomized order using the Bead-Beat Micro AX Gravity Kit (A\&A Biotechnology, Poland) according to manufacturer's instructions. Prior to extraction, samples were lysed in LSU buffer supplemented with Lysozyme (4000 U) and Mutanolysin (50 U), and incubated at $50^{\circ} \mathrm{C}$ for $20 \mathrm{~min}$. The concentration and purity of extracted DNA were determined using a NanoDrop ND-1000 spectrophotometer and normalized to $10 \mathrm{ng} / \mu \mathrm{l}$. High throughput sequencing based 16S rRNA gene amplicon (V3region) sequencing was carried out on an Illumina NextSeq platform as previously described (30).

The raw dataset containing pair-ended reads with corresponding quality scores were merged and trimmed using fastq_mergepairs and fastq_filter scripts implemented in the USEARCH pipeline as described previously (30). Purging the dataset from chimeric reads and constructing zero radius Operational Taxonomic Units (zOTU) was conducted using UNOISE. The Greengenes (13.8) 16S rRNA gene collection was used as a reference database. Quantitative Insight Into Microbial Ecology (QIIME) open source software package (v2019.7.0) was used for subsequent analysis steps (31). Alpha diversity measures: observed species (number of zOTUs) and Shannon diversity indices were computed for rarefied OTU tables (10,000 reads/sample) using the alpha rarefaction workflow. Differences in alpha diversity were determined using a t-test-based approach employing the non-parametric (Monte Carlo) method (999 permutations) implemented in the compare alpha diversity workflow. Principal Coordinates Analysis $(\mathrm{PCoA})$ plots were generated with the Jackknifed Beta
Diversity workflow based on 10 distance metrics calculated using 10 sub-sampled OTU tables. The number of sequences taken for each jackknifed subset was set to $85 \%$ of the sequence number within the most indigent sample $(\sim 10,000)$. Community differences (beta-diversity) were revealed by weighted and unweighted Unifrac distance metrics visualised as Principle Coordinate Analysis (PCoA) plots. Permutational Multivariate Analysis of Variance (PERMANOVA) and Non-parametric microbial interdependence test (NMIT) were used to evaluate group differences based on weighted and unweighted UniFrac distance matrices. Taxa-level differences were assessed using longitudinal feature-volatility analysis and analysis of composition of microbes (ANCOM).

\section{Statistical Analysis}

Data were analysed using general linear model (GLM) using IBM SPSS Statistics 28. For each separate probiotic mixture (BBE or LB), the effects of probiotic supplementation and parasite infection, and their interaction, were compared to control-fed animals using a separate factorial analysis. The model included infection status, probiotic supplementation and sex as fixed factors, together with their first-order interactions. Sex was removed from the model when not significant. For analysis of ELISA data, time was included as an additional fixed factor to account for repeated measurements. Assumptions of normality were checked through inspection of histogram plots and Shapiro-Wilk and Kolmogorov-Smirnov tests of GLM residuals, and data that did not conform to normality was transformed with either square-root or $\log _{10}$ transformations prior to analysis. Significance was taken at $p<0.05$, and a trend at $p<0.1$.

\section{RESULTS}

\section{Effects of Probiotics on the Intestinal Environment and $\mathrm{O}$. dentatum Infection Levels}

Eight - ten week old pigs $(n=48)$ were divided into three groups (Supplementary Figure 1). 16 pigs received only the basal control diet (based on ground barley and soybean meal) throughout the study, 16 pigs received the basal diet supplemented with a mixture of Bacillus amyloliquefaciens $B$. subtilis, and Enterococcus faecium (hereafter BBE), and 16 pigs received the basal diet supplemented with a mixture of Lactobacillus rhamnosus LGG and Bifidobacterium animalis subsp. Lactis BB-12 (hereafter LB). The BBE mixture was selected based on its development specifically to improve gut health in pigs, whilst LB was chosen as it contained two wellstudied probiotic strains that have been shown to induce immunomodulatory activity in pigs $(22,32)$. Within each dietary group, following a 14 day acclimatization period, half the pigs $(n=8)$ were either trickle-infected throughout the study with $O$. dentatum larvae $(n=24)$, or remained uninfected $(n=24)$.

To explore the effects of probiotics on the response to helminth infection, we quantified the effect of probiotic 
supplementation on intestinal physicochemical parameters and parasite establishment and development. We first assessed the concentrations of SCFA and D-lactic acid in the proximal colon (Figure 1A), with a separate analysis conducted for the two different probiotic-supplemented groups, relative to those with no supplementation. Acetic and propionic acid concentrations were unaffected by either infection or probiotic supplementation. $O$. dentatum infection significantly increased n-Butyric acid levels $(p<0.05)$ in pigs fed either the control diet alone or in those supplemented with BBE. However, there was no effect of $O$. dentatum when analysing LB-supplemented pigs, indicating that the effect of infection varied according to specific probiotic intake (Figure 1A). Total SCFA levels were not different between any of the groups. In contrast, D-lactic acid levels were significantly increased by LB supplementation, and tended also to be increased by BBE supplementation $(p=0.08)$, independently of infection (Figure 1A). Neither probiotic supplementation nor infection influenced the $\mathrm{pH}$ in the jejunum or ileum (data not shown), or the caecum or proximal colon (Figure 1B). However, infection resulted in a lower $\mathrm{pH}$ in the distal colon $(p<$ 0.05; Figure 1B).

Supplementation with either of the probiotic mixtures did not significantly influence infection levels or parasite infection kinetics, with average worm numbers (adult and larval $O$. dentatum) of $15,843 \pm 2,128$ and $17,425 \pm 2,185$ (mean \pm SEM) for pigs fed $\mathrm{BBE}$ and $\mathrm{LB}$ probiotics respectively, compared to $18,455 \pm 2,598$ for the control-fed group (Figure 2). Moreover, probiotic supplementation had no effect on worm length (data not shown) nor egg production; with similar eggs per gram faeces (EPG) scores observed for all diet treatment groups. In addition, no significant differences in body weight gain were observed between the dietary treatment/ infection groups, with all pigs gaining weight consistently over the course of the experimental period (data not shown).

\section{O. dentatum Infection Changes the Response of the Fecal Microbiota to Probiotic Supplementation}

To examine if the two probiotic mixtures and/or O. dentatum affected the composition of the prokaryotic GM, we conducted longitudinal sampling and analyses of faeces over the course of the study. Across the time period, $\alpha$-diversity remained stable in pigs with no probiotic supplementation, regardless of whether they were infected with $O$. dentatum or not, with no significant differences in Faith phylogenetic diversity (PD) (Figure 3A). In contrast, in both uninfected and $O$. dentatum-infected pigs, BBE or LB supplementation tended to increase the Faith PD over time (indicative of a more diverse microbiota at the end of the study than at the start), ( $p=0.065$ for LB in infected pigs; $p=0.05$ in other cases) (Figure 3A).

There was also a significant shift in $\beta$-diversity in the fecal GM as a result of probiotic supplementation, but this was dependent on infection status. Non-parametric microbial interdependence testing (NMIT) indicated that infected pigs fed BBE differed in $\beta$-diversity from infected pigs without probiotic supplementation $(p<0.05$; Figure $3 \mathbf{B})$. However, this was not the case for uninfected pigs $(p=0.26$; Figure 3B). A contrasting effect was observed for LB, where uninfected pigs fed LB diverged from uninfected pigs without probiotic supplementation $(p<0.05)$, yet infected pigs fed LB did not differ from infected pigs without LB (Figure 3C). In the absence of probiotic supplementation, infection did not influence $\beta$ diversity. Analyses on pooled data revealed a similar story, with both LB and BBE-fed pigs (independent of infection
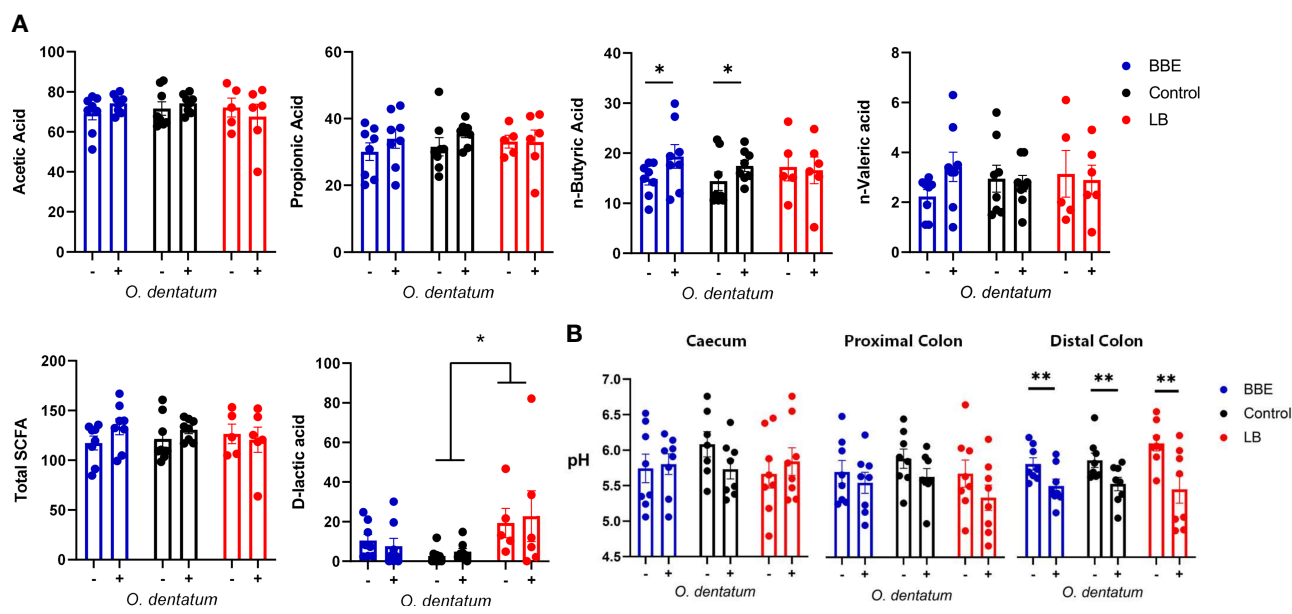

FIGURE 1 | Effect of probiotics and Oesophagostomum dentatum infection on the intestinal environment. (A) Microbial metabolite (short-chain fatty acids and Dlactic acid) concentrations from proximal colon digesta after 28 days of $O$. dentatum infection, in pigs fed a control diet or a diet supplemented with either a mixture of Enterococcus faecium and Bacillus sp. (BBE), or LGG and Bb12 (LB). Metabolite concentrations are expressed in mmol/kg wet sample. (B) pH of digesta sampled throughout the intestinal tract. Statistical analysis was conducted separately for each probiotic treatment, using a GLM analysis comparing the effect of probiotic supplementation and infection (and their interaction) to the control-diet groups (no probiotics). Data presented as means \pm SEM ( ${ }^{\star} p \leq 0.05$, ${ }^{*} p \leq 0.005$, by GLM). $n=8$ pigs per treatment group. 


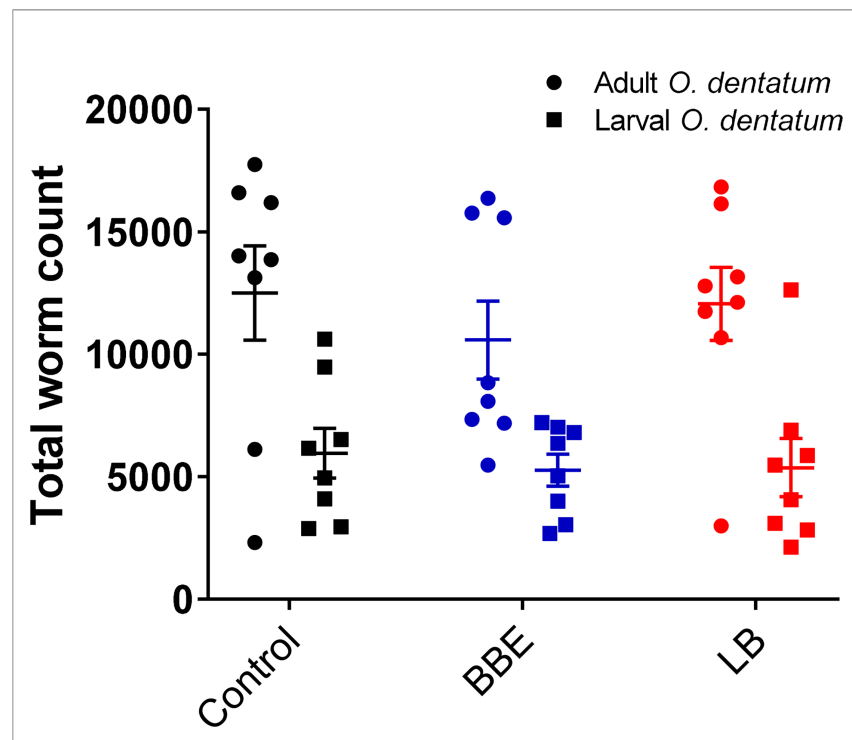

FIGURE 2 | Oesophagostomum dentatum burden is not affected by probiotic treatment. O. dentatum adult and larval worm burdens, at day 28 post-infection in pigs fed a control diet or a diet supplemented with either a mixture of Enterococcus faecium and Bacillus sp. (BBE), or LGG and Bb12 (LB). Data presented as means \pm SEM. $n=8$ pigs per treatment group.

status) significantly diverging from control-fed pigs $(p<0.05)$, whereas infection status (independent of probiotic supplementation) had no effect (Supplementary Figure 2). Taken together, these data suggest that over the course of the seven week experiment, both BBE and LB probiotics induced modest but significant changes in the composition of the fecal microbiota, yet these probiotic-induced changes were further influenced by concurrent $O$. dentatum infection.

To explore which bacterial taxa were responsible for the divergence between probiotic-fed pigs and their respective controls without probiotics, Feature Volatility analysis was performed. Within uninfected pigs, seven taxa were enriched in pigs receiving BBE compared to those that did not, most notably the Bifidobacterium genus, whilst a single family (Succinivibrionaceae) belonging to the Proteobacteria phylum decreased in abundance (Figure 3D). However, in infected pigs fed BBE, relative abundance of Bifidobacterium spp. was lower compared to infected pigs without $\mathrm{BBE}$, indicating that the infection abrogated the probiotic-stimulated increase in Bifidobacteria. Turicibacter sp., a genus we have previously observed to be enriched in the colon of pigs infected with Ascaris suum (28), was elevated in infected pigs fed BBE compared to uninfected controls. Similarly, the effects of LB varied depending on infection status (Figure 3D). In uninfected pigs, only two taxa differed between LB-fed pigs and control-fed pigs without LB. In contrast, relative to the control group (uninfected pigs without probiotics), infected pigs fed LB had higher relative abundance of several members of the Firmicutes phylum including two Lactobacillus species, as well as Mitsuokella multacida, a putative butyrate producer and beneficial microbe (33). Collectively, these data suggest that BBE tended to enrich beneficial bacteria such as Bifidobacterium in feces over the course of the experiment in uninfected pigs, but these effects were reversed in $O$. dentatuminfected pigs. Conversely, LB tended to enrich beneficial bacteria such
A

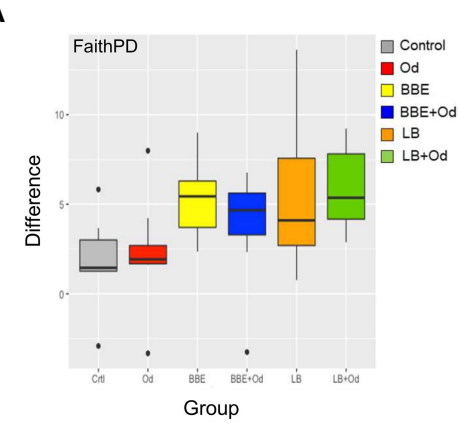

B

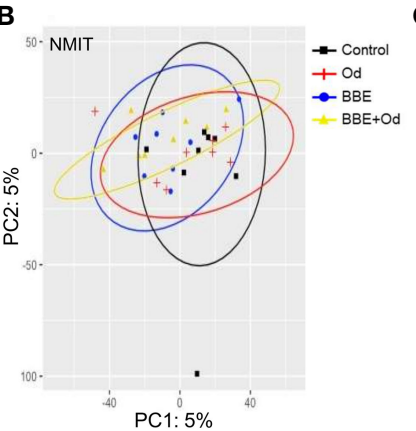

C

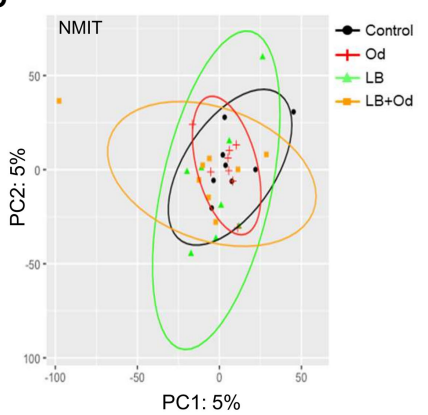

D

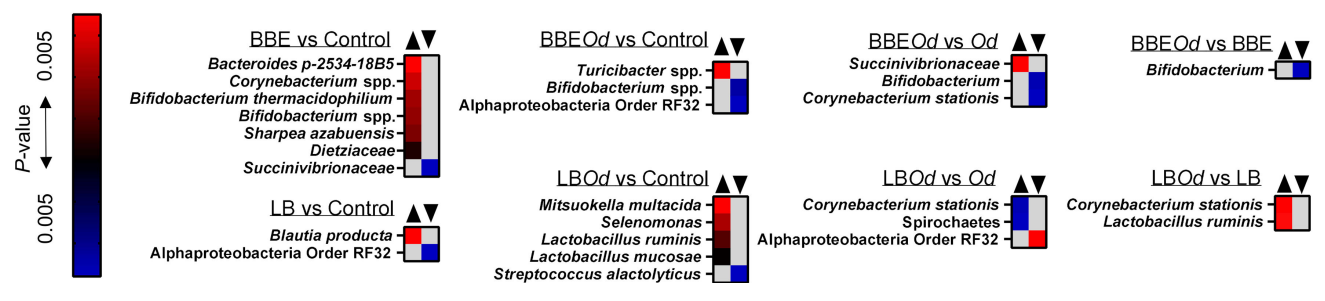

FIGURE 3 | Probiotics modulate the fecal gut microbiota over time. (A) Alpha-diversity (Faith PD) in feces samples over time [pairwise comparison between day -7 and 28 post-infection (p.i.)]. Pigs were either uninfected or infected with $\mathrm{O}$. dentatum (Od) and fed a control diet or a diet supplemented with either a mixture of Enterococcus faecium and Bacillus sp. (BBE), or LGG and Bb12 (LB). (B, C) NMIT PCoA showing effect of infection and diet in pigs fed BBE (B) or LB (C) from day -7 to day 28 p.i. (D) Taxa where abundance was significantly altered in feces across the course of the experiment as a result of infection or diet, as identified by Feature Volatility Analysis. $n=8$ pigs per treatment group. 
as Lactobacillus more strongly in the feces of $O$. dentatum-infected pigs than uninfected pigs. Thus, $O$. dentatum alone did not change the composition of the fecal microbiota over the course of the study, but instead modulated the effect of BBE and LB in two distinct ways, indicating a complex interaction between probiotics and the parasitic infection.

\section{Probiotics and O. dentatum Infection Interact to Change the Intestinal Microbiota in a Site-Specific Manner}

We next investigated how infection and/or probiotics influenced the microbiota composition throughout the intestinal tract. Similarly to the longitudinal fecal samples, $\alpha$ diversity (Faiths PD) was increased by both BBE and LB in comparison to control pigs, mainly in the distal colon, with a comparable effect in both infected and uninfected pigs $(p=$ 0.093 for infected pigs fed LB; $p<0.05$ for other comparisons; Figure 4). Notably, $O$. dentatum infection was also associated with increased $\alpha$-diversity in the distal colon (Figure 4 and Supplementary Table 2). Effects of infection and treatment were not as pronounced in the other gut segments (Figure 4 and Supplementary Table 2).

Analysis of unweighted Unifrac distance metrics showed that, in the absence of probiotic supplementation, the only intestinal site where $O$. dentatum infection significantly changed $\beta$-diversity, relative to uninfected pigs, was the proximal colon (the predilection site of the worms) ( $p<0.05$ by PERMANOVA; Figure $4 \mathrm{~B}$ ). $\beta$-diversity in the gut was also considerably altered by probiotic supplementation. Changes were primarily observed via unweighted Unifrac analysis, indicating that most differences were driven by low-abundance species. In uninfected pigs, BBE supplementation altered $\beta$-diversity compared to pigs without probiotic supplementation in the ileum, caecum and both proximal and distal colon ( $p=0.096$ for caecum, $p<0.05$ for all other segments by PERMANOVA; Figure 4C and Supplementary Table 3). However, this effect was less evident when the BBEsupplemented pigs were infected with $O$. dentatum. In these animals, supplementation with BBE resulted in no significant difference in $\beta$-diversity in the ileum or caecum relative to control pigs (uninfected and without probiotics). Furthermore, lesser (albeit still significantly different) changes were observed in the colon between control pigs and infected pigs receiving BBE (Figure 4C and Supplementary Table 3). Thus, infection appeared to attenuate the BBE-induced changes in GM composition.

$\mathrm{LB}$ also tended to alter $\beta$-diversity in the jejunum and caecum, with similar changes in both uninfected and infected pigs $(p<0.1$ by PERMANOVA; Figure 4C and Supplementary Table 4). LB had a stronger effect in the colon (both proximal and distal). Here, significant divergence was observed between control and LB-fed pigs, regardless of infection status $(p<0.05$ by PERMANOVA). However, within LB-fed pigs, infected pigs were significantly diverged from uninfected pigs with infected pigs clustering closer to the control animals $(p<0.05$ by PERMANOVA; Figure 4C and Supplementary Table 4), again indicating that infection tended to limit the modulatory effects of the probiotics on the GM.
We attempted to identify specific taxa responsible for the differences between treatment groups, however ANCOM analysis yielded no significant differences in any gut segment $(p>0.05)$. Thus, the changes in the GM community within the gut segments appeared to derive from the cumulative effect of subtle alterations across multiple taxa, rather than substantial alterations in the abundance of precise bacterial species.

\section{Both Probiotics and O. dentatum Infection Influence Peripheral and Local Immune Function}

We next assessed how probiotic supplementation modulated the development of the systemic and mucosal response to $O$. dentatum infection. Serum IgA and $\operatorname{IgG}_{1}$ antibody levels were measured weekly until day 28 p.i. All pigs were sero-negative for $O$. dentatum prior to study start at day 0 . Infection with $O$. dentatum resulted in increased $O$. dentatum-specific antibody titres compared to uninfected pigs (Figure 5A). Both IgA and $\mathrm{IgG}_{1}$ antibody titre levels increased from day 7 through until day 28 p.i. There was a significant interaction between time and LB probiotics at day 21 p.i., whereby LB-fed infected pigs had higher IgA levels compared to the other infected groups $(p<0.005)$, however this difference was not apparent at other time points. BBE probiotics did not influence IgA titres, and there was no effect of probiotic supplementation on $\mathrm{IgG}_{1}$ titres.

Analysis of CLN lymphocyte populations revealed a significant interaction between BBE probiotic supplementation and $O$. dentatum infection. In control-fed pigs, infection increased the percentage of T cells $(p<0.01)$, and reduced the percentage of B-cells $(p<0.05)$ resulting in an altered T-cell/Bcell ratio (Figure 5B). However, this effect was not apparent in infected pigs fed the BBE probiotics, with the T-cell/B-cell ratio equivalent to uninfected pigs, indicating that $O$. dentatuminduced alterations in lymphocyte populations were attenuated in these animals (Figure 5B). In contrast, LB probiotic supplementation did not have this modulatory effect, with no significant interaction and only a main effect of infection in analysis of both T-cell and B-cell populations (Figure 5B). Analysis of other cell populations within CLN or PBMC, namely $\mathrm{CD} 3{ }^{+} \mathrm{CD} 4^{+}$helper and $\mathrm{CD} 3^{+} \mathrm{CD} 8^{+}$cytotoxic $\mathrm{T}$ cells, or monocytes, showed no significant effects of either diet or infection (data not shown).

To assess functional cellular immune responses in peripheral and lymphoid tissues, PBMCs and CLN cells were stimulated with LPS or PHA, respectively, and cytokine secretion quantified. Infection did not consistently change the cytokine secretion pattern (Figure 6). In contrast, BBE supplementation substantially modulated cytokine profiles, although the effect was dependent on infection status. There was an interaction $(p<$ 0.05 ) between probiotics and infection on mitogen-induced TNF $\alpha$ secretion from CLN cells, with BBE supplementation significantly reducing TNF $\alpha$ production in uninfected pigs, but not in infected animals. In contrast, IL-10 production tended to be enhanced by BBE in both infected and uninfected pigs ( $p=$ 0.06 for main effect of probiotic supplementation; Figure 6A). 


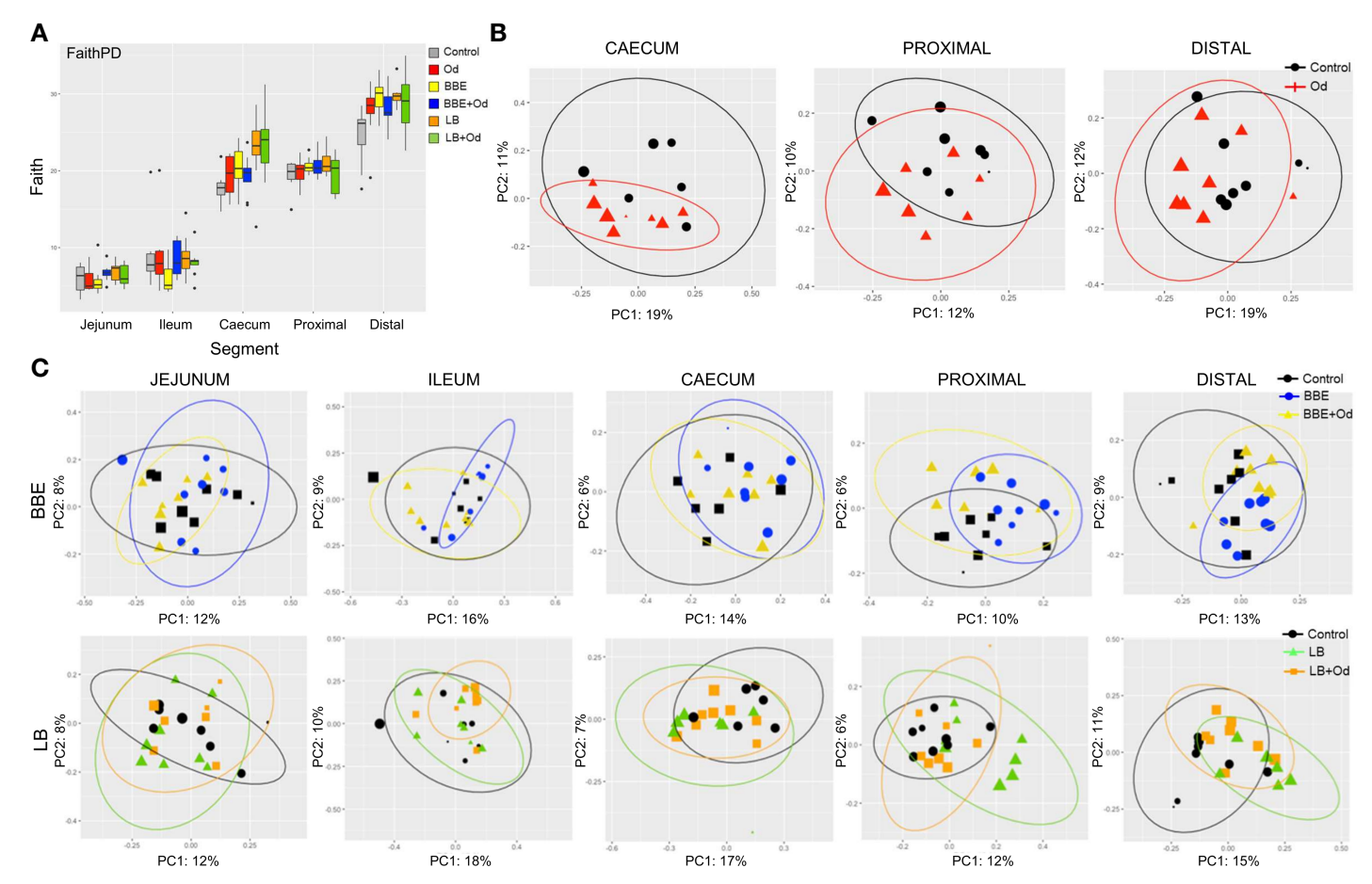

FIGURE 4 | Probiotics and parasite infection modulate the gut microbiota in different gastrointestinal compartments. (A) Alpha-diversity (Faith PD) in different gut segments at day 28 post-infection. Pigs were either uninfected or infected with $O$. dentatum (Od), and fed a control diet or a diet supplemented with a either mixture of Enterococcus faecium and Bacillus sp. (BBE), or LGG and Bb12 (LB). P-values are shown in Supplementary Table 1. n=8 pigs per treatment group. Color of symbols is according to group, size is according to the alpha index Shannon. (B) Unweighted PCoAs for pairwise comparisons of uninfected and Oesophagostomum dentatum (Od)-infected pigs fed only the control diet (no probiotics) in the caecum and proximal and distal colon. (C) Unweighted PCoAs for pairwise comparisons of uninfected and $O$. dentatum $(\mathrm{Od})$-infected pigs fed only the control diet (no probiotics), or a diet supplemented with either a mixture of Enterococcus faecium and Bacillus sp. (BBE), or LGG and Bb12 (LB).

In PBMCs, BBE significantly suppressed LPS-induced IL- $1 \beta$ in both uninfected and infected pigs ( $p<0.05$; Figure 6B), with a similar tendency for IL-10 secretion ( $p=0.06$; Figure 6B). TNF $\alpha$ followed the same pattern but the differences were not significant (Figure 6B). There was an interaction $(p<0.05)$ between probiotics and infection for IL- 6 production, with secretion reduced in uninfected pigs fed with $\mathrm{BBE}$, but tended to be enhanced in infected pigs (Figure 6B). The effects of LB probiotics were less apparent. LB supplementation resulted in lower $(p<0.05)$ TNF $\alpha$ secretion from CLN cells, independently of infection status, but there were no effects on the other cytokines measured in either CLN or PBMC (Supplementary Figure 3). As the cellular composition of PBMC was not significantly affected by probiotic supplementation, this suggests that the functional activity of these cells was specifically altered in response to probiotics. Collectively, these data suggest that BBE probiotics have an anti-inflammatory effect in the absence of parasite infection. However this effect was modulated in infected pigs. Whereas IL-1 $\beta$ was strongly suppressed in PBMC from both uninfected and infected animals receiving $\mathrm{BBE}$, the effect on other cytokines such as IL-6 appeared to be influenced by the parasitic infection, with the suppressive effect less evident in infected pigs. These data suggest that concurrent helminth infection may restrict the antiinflammatory properties of BBE probiotics.

\section{Probiotics Attenuate O. dentatum-Induced Inflammatory Gene Expression in the Proximal Colon}

To explore in more detail if the dietary probiotics modulated local host immune responses, we investigated changes in gene expression in the proximal colon during O. dentatum infection. A panel of genes was selected to represent Th1-, Th2- and regulatory immune responses, as well as mucosal barrier and innate immunity-related genes. Principal component analysis (PCA) of the relative expression of all genes analysed in the proximal colon illustrated a marked effect of $O$. dentatum infection (Figure 7A), and a lesser influence of probiotic supplementation (Figure $\mathbf{7 B}$ ). In the absence of probiotic supplementation, there was a prototypical type- 2 polarised immune gene expression profile in the proximal colon of pigs infected with $O$. dentatum, relative to uninfected animals. Infection with $O$. dentatum significantly increased expression of IL4, IL13, ARG1, CCL17 and CCL26, with a concurrent trend for down-regulation of the expression of Th1-related genes such as IL8 (Figure 7C and Supplementary Table 5). In addition, 

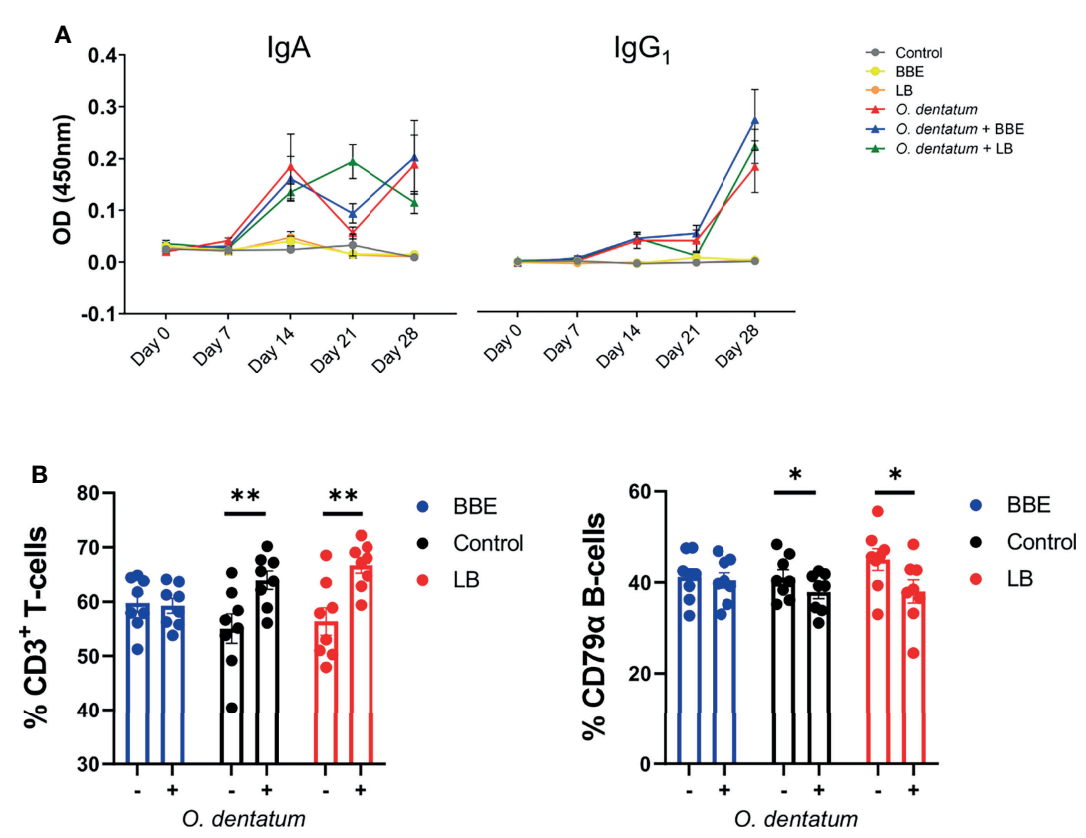

FIGURE 5 | Systemic and peripheral immune responses elicited towards Oesophagostomum dentatum infection. (A) O. dentatum specific IgA and IgG 1 serum antibody production over the 28 days of infection in pigs fed a control diet or a diet supplemented with either a mixture of Enterococcus faecium and Bacillus $\mathrm{sp}$. (BBE), or LGG and Bb12 (LB). (B) Flow cytometric analysis of ileo-caecal lymph node cells obtained at day 28 post-infection. \% CD3+ T cells and \% CD79 $\alpha$ B-cells. Statistical analysis was conducted separately for each probiotic treatment, using a GLM analysis comparing the effect of probiotic supplementation and infection (and their interaction) to the control-diet groups (no probiotics). Data presented as means $\pm \mathrm{SEM}\left({ }^{\star} p<0.05,{ }^{\star \star} p<0.01\right.$ by GLM). $n=8$ pigs per treatment group.

increased expression of mucosal barrier-related genes, such as RETNLB, FFAR2, and DCLK1, and innate immune genes such as IL6, C3 and PTGS2 (encoding cyclooxygenase-2) were also observed in infected, control-fed pigs (Figure 7C and Supplementary Table 5).

We noted a moderately enhanced Th1 polarization as a result of probiotic supplementation. Both probiotic treatments increased the expression of IL8, IL12B and INOS in both uninfected and $O$. dentatum-infected animals. LB supplementation also significantly increased IFNG expression (Figure 7C and Supplementary Table 5), as well as CXCL10 expression but only in males $(p<0.05$ for interaction between sex and LB supplementation).

Strikingly, in O. dentatum-infected pigs, BBE supplementation markedly attenuated the helminth-induced increases in gene expression relative to control-fed animals. In BBE-fed pigs, Th2 genes were still up-regulated as a result of helminth infection, but to a lesser degree compared to $O$. dentatum infected pigs fed only the control diet (Figure 7C). For genes where there was a significant interaction $(p<0.05)$ between BBE supplementation and infection, in every case this resulted in significant down-regulation of expression in infected, BBE-fed pigs compared to infected, control-fed pigs (Supplementary Table 5). This included key Th2 and epithelial/mucosal barrier related genes, including those coding for the short-chain fatty acid receptor FFAR2, the epithelial cell kinase and tuft cell marker DCLK1 the interleukin-4 receptor IL4, and the eosinophil chemoattractant CCL26 (Figure 7D). Moreover, the helminth-induced expression of other immune related genes such as TNF, CTLA4 and PLA2G4A was significantly attenuated by BBE supplementation (Figure 7D). This was evident in PCA analysis which showed that $O$. dentatuminfected pigs administered BBE clustered closer to uninfected control pigs than $O$. dentatum-infected pigs without probiotic supplementation, suggesting that the response to infection was muted in these animals, and that BBE acted to restrain the localized inflammatory response to the parasite (Figure 7E). A similar pattern was evident in infected pigs with LB supplementation, but the effect was less pronounced, with the immune gene profile more closely resembling that of $O$. dentatum-infected pigs fed the control diet (Figure 7E). However, we did note a trend $(p<0.1)$ for interactions between infection and LB supplementation for the expression, of ARG1, TLR3, IL1B, and CTLA4, with the infection-induced expression of these genes being attenuated to some extent by LB (Figure 7C and Supplementary Table 5). Thus, probiotic supplementation (most primarily with BBE) acted to attenuate parasite-induced, type-2 biased inflammatory responses in the colon.

\section{DISCUSSION}

The beneficial effect of probiotics on health and control of bacterial infections is well-documented, however the potential interactions of probiotics with helminth infection and the 

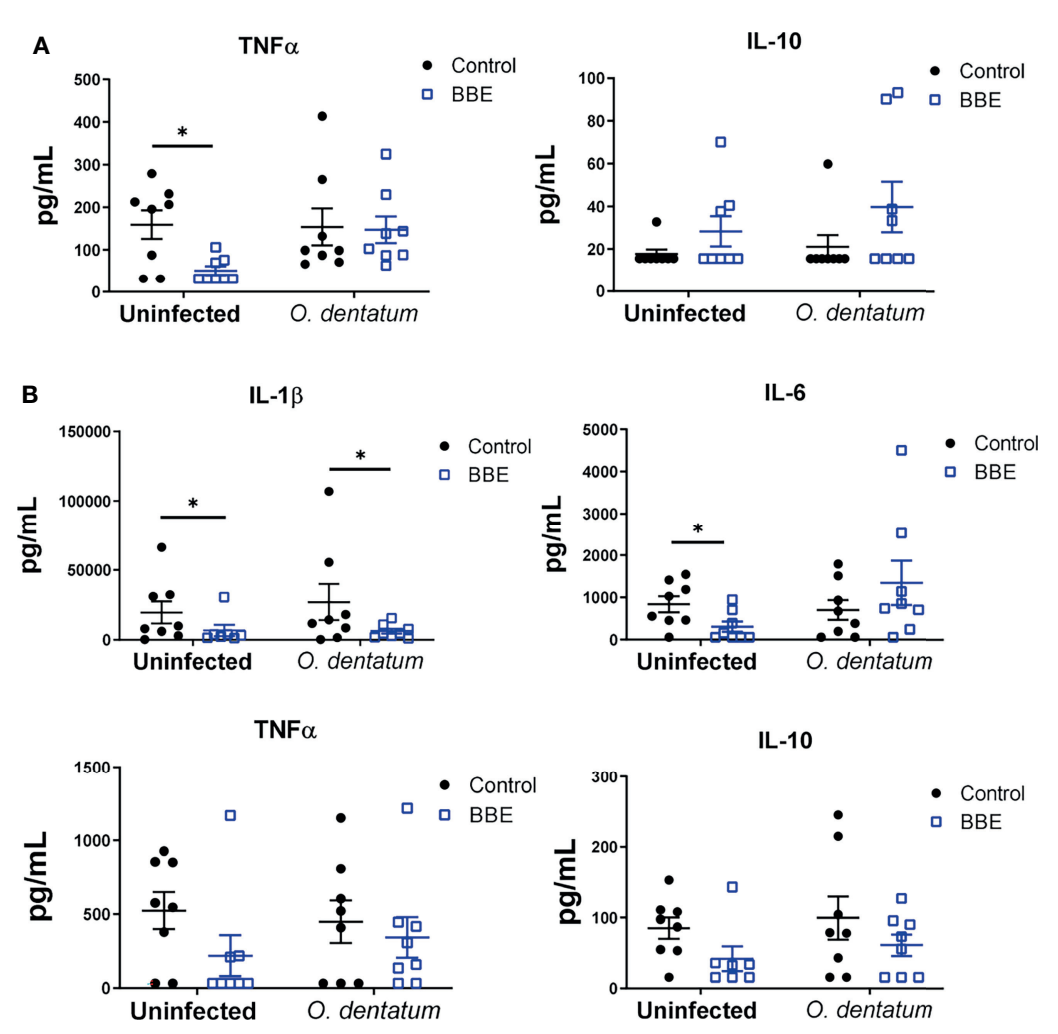

FIGURE 6 | Ex vivo cytokine secretion is modulated by probiotics and Oesophagostomum dentatum infection. (A) Phytohaemagglutinin-induced secretion of TNF $\alpha$ and IL-10 in ileal-cecal lymph node cultures. Pigs were either uninfected or infected with 0 . dentatum for 28 days, with or without supplementation of a mixture of Enterococcus faecium and Bacillus sp. (BBE). (B) LPS-induced secretion of IL-1 $\beta, I L-6$, TNF $\alpha$ and IL-10 in peripheral blood mononuclear cells from pigs infected with 0 . dentatum for 28 days or uninfected pigs, with or without supplementation of BBE. * $p<0.05$ by GLM analysis. $n=8$ pigs per treatment group.

mechanisms by which they can influence mucosal immune responses is not well understood. Here, we assessed for the first time how probiotic intake could modulate worm burdens, immune function and GM composition during a colonic helminth infection in swine.

We found that probiotics (BBE in particular) were capable of suppressing ex vivo inflammatory cytokine production and attenuating the host mucosal immune responses elicited in response to infection. Neither probiotic mixture modulated the establishment or infection kinetics of $O$. dentatum. However, both mixtures appeared to beneficially modulate the intestinal microbiota composition, as evidenced by increased bacterial diversity in both fecal and large intestinal samples. Interestingly, we noted that these effects were to some extent modulated by $O$. dentatum infection, suggesting a novel interaction of parasite infection on probiotic activity. Furthermore, we observed attenuation of the prototypical type2 inflammation induced by $O$. dentatum by BBE probiotics.

$O$. dentatum infection is highly prevalent in pigs worldwide. dietary prebiotics that can modulate host GM, such as inulin, have been shown to be highly effective in reducing parasite burdens, however our results here show that supplementation of specific probiotic strains did not have an anti-parasitic effect. The mode-of-action of prebiotics against $O$. dentatum is hypothesized to result from a selective enrichment of lactic acid producing bacteria, and production of GM-derived metabolites such as SCFA, which lower the colon $\mathrm{pH}$ and create an inhospitable environment for helminths (19). Despite an increase in D-lactic acid induced by LB, we did not observe changes in gut $\mathrm{pH}$ (or total SCFA levels) as a result of either probiotic mixture. Thus, the administration of certain probiotic bacteria was insufficient to have an anthelmintic effect, although associated changes in the immune system or GM may still markedly impact gut health. A number of previous studies in murine models have shown conflicting results about whether probiotics can exert anthelmintic activity against parasites such as Schistosoma mansoni, Trichinella spiralis or Trichuris muris, with some reports of reduced parasite burdens following probiotic intake, and also reports of no effect or even significantly increased infection (34-36). There may be numerous reasons for these discrepancies, but it is known that the composition of the basal diet (e.g. fibre contents) may play a role in the activity of probiotics, and therefore the combination of prebiotic dietary components and probiotics (i.e. 'synbiotics') should be further investigated to optimize the potential antiparasitic activity of probiotic bacteria.

Helminth infection is typically associated with a rise in antibody secretion and the initiation of a characteristic Th2 


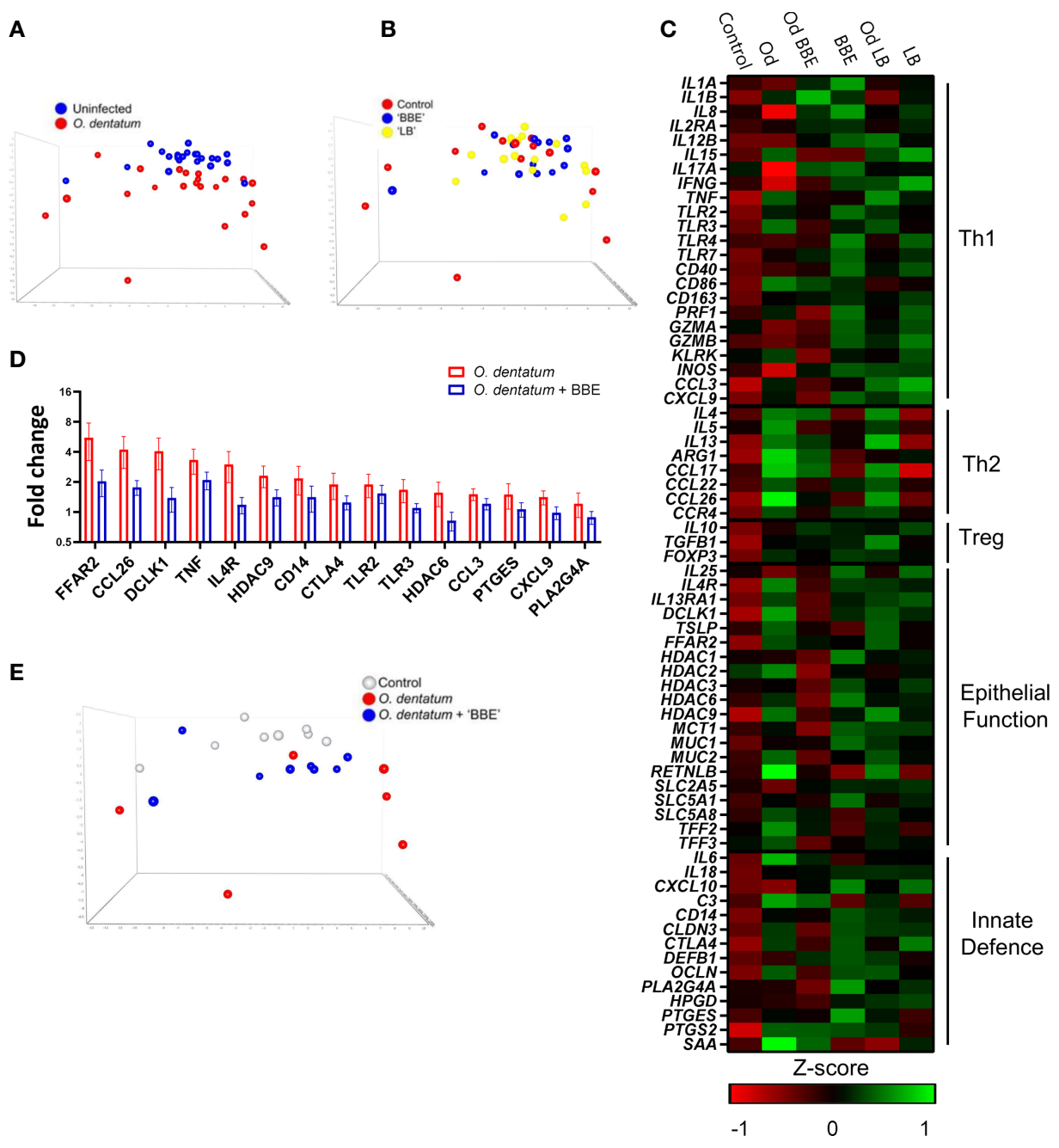

FIGURE 7 | Probiotics and Oesophagostomum dentatum infection alters immune gene expression profiles. (A, B) Principal component analysis of immune gene expression in the proximal colon at day 28 post-infection (p.i.) as a result of $O$. dentatum infection (A) or diet supplementation with probiotic mixtures Enterococcus faecium and Bacillus sp. (BBE), or LGG and Bb12 (LB) (B). (C) Expression of genes involved in different biological function as a result of O. dentatum infection (Od), BBE or LB supplementation, or $O$. dentatum infection combined with BBE or LB supplementation. The control group received no infection or probiotic treatment. Data presented as Z-scores of relative gene expression data. (D) Fold changes in expression of genes from proximal colon tissue significantly altered ( $p<0.05$ ) by the interaction of Oesophagostomum dentatum infection and dietary supplementation with BBE, in comparison to control-fed, 0 . dentatum-infected controls. $\mathrm{n}=6-8$ pigs per treatment group. (E) Principal component analysis showing immune gene expression in the proximal colon at day 28 p.i. in control pigs (no infection or probiotics), O. dentatum infection without probiotics, and O. dentatum with BBE supplementation.

immune response. Similarly to Andreasen et al. (24) we observed a type-2 immune response in control-fed pigs infected with $O$. dentatum, with increased antibody secretion, peripheral $\mathrm{T}$ cell activation, and type- 2 immune gene expression profiles in the proximal colon confirming an active host immune response was elicited. Interestingly, infected pigs fed BBE probiotics exhibited a reduction in epithelial immune genes, such as TSLP, IL4R and FFAR2, compared to the O. dentatum-infected pigs fed only the control diet. In addition, BBE treatment alone tended to reduce expression of key Th2 immune genes, such as IL4, IL5 and CCL26, and appeared to diminish the parasite-induced increase in the expression of these genes in infected pigs fed BBE. Together, this suggests that the typical polarised helminth- mediated Th2 immune response is attenuated by the supplementation of Bacillus spp. and E. faecium-based probiotics. This attenuation of prototypical helminth-induced immune response has been observed previously in A. suuminfected pigs fed L. rhamnosus LGG (23). Jang et al. (23) reported reduced $\operatorname{IgG}_{2}$ antibody titres and reduced expression of IL13, eosinophil peroxidase EPX, and CCL26 in A. suum-infected pigs supplemented with LGG. The observed suppression of Th2 and epithelial gene expression profiles in this study may have been the result of the probiotics exerting a regulatory effect to maintain intestinal immune homeostasis.

We observed that probiotic supplementation appeared to significantly alter the intestinal microbiota, with both mixtures 
(BBE and $\mathrm{LB}$ ) improving the microbial diversity and richness over the course (day 28 post-infected compared to 7 days preinfection) of the study and at different segments of the intestinal tract. PERMANOVA analysis confirmed that probiotic supplementation did have a modulatory effect on the microbiota, although the changes could not be ascribed to specific taxa. The modest impact of probiotics on the composition of the GM appears to be in keeping with several studies that reported minor compositional alterations as a result of supplementation with a range of probiotic strains $(7,37)$. Interestingly, both probiotic mixtures induced subtle alterations to SCFA and lactic acid levels present in intestinal digesta, suggesting that even with limited changes in the GM, potentially beneficial outcomes to intestinal health can still be achieved, as was evident by the modulation of intestinal immune gene expression profiles.

To our knowledge this is the first time the porcine GM has been characterised during O. dentatum infection. Consistent with previous observations in pigs infected with T. suis $(20,21), O$. dentatum infection altered $\beta$-diversity in the caecum and colon. However, unlike T. suis, this modulation did not appear to be associated with defined bacterial taxa, and significant changes were not observed in faeces or the small intestine. This suggests that $O$. dentatum infection had a localised impact on the GM without inducing changes throughout the intestinal tract. The most striking observation was the apparent ability of $O$. dentatum to suppress the changes in the GM brought about by probiotics that were observed in uninfected pigs. Thus, concurrent parasitic infections, which are common in livestock and humans in developing countries, may be a previously unappreciated factor influencing the health benefits of dietary probiotics.

The mechanisms by which probiotics alter the response to helminth infection requires further investigation. Various modesof-action have been proposed for the health benefits of probiotic bacteria. Probiotics may adhere to intestinal epithelial cells and thereby prevent the attachment of potentially pathogenic bacteria such as E. coli, as well as inducing mucus production and the stimulation of antimicrobial peptides (38). Furthermore, probiotics may regulate inflammatory responses by binding to PRRs on immune cells and promoting secretion of IL-10 or TGF- $\beta$, which can suppress inflammatory cytokine production (39). Moreover, probiotics such as LGG have previously been shown to promote Th1 responses in pigs, and the Th1-stimulating properties of probiotics has been suggested to underlie the ability of probiotics to suppress symptoms of allergies in humans and animal models $(39,40)$. Indeed, our gene expression data in the colon indicated a modest Th1-polarizing effect of both probiotic mixtures in the absence of infection, suggesting that host PPRs recognize the bacteria and respond with production of type-1 cytokines and innate immune mediators that are typically produced in response to TLR or NOD receptor binding $(41,42)$. Probiotics have also been shown to induce regulatory responses that can alleviate inflammation during pathogen challenge in pigs (43), and thus the attenuation of the helminth-induced type- 2 response may then derive from the ability of the probiotic bacteria to restore homeostasis in the face of acute pathogen-driven inflammation.
Interestingly, we observed that $\mathrm{BBE}$ appeared to be more efficient than LB in modulating host immune responses, which may reflect the inclusion of porcine-derived strains in the BBE mixture. Further experiments to identify specific immune cell populations that are modulated by probiotics (e.g., dendritic cells, intra-epithelial lymphocytes) are now highly warranted, as are studies in other porcine pathogen infection models to see how the outcome of infections that promote type- 1 immunity (e.g. viral infections) is altered in response to probiotic intake. Moreover, more targeted investigations with integrated analytical techniques to further determine the trilateral associations between worms, specific bacterial taxa and immune molecules are also a high priority.

In conclusion, we show here that probiotics, in particular the strains Bacillus amyloliquefaciens, B. subtilis, and Enterococcus faecium, do not appear to directly affect worm establishment and development but do regulate inflammatory responses and attenuate host mucosal immune function during $O$. dentatum infection, which may serve to regulate host intestinal function and maintain immune homeostasis. This probiotic-mediated regulation of host immune responses is also indicative of the ability of probiotics to potentially dampen Th2-mediated pathology as a result of, for example, food allergies (44-46). Moreover, the ability of these probiotic strains to attenuate pathogen-induced inflammatory responses may have relevance for dietary interventions that seek to maintain intestinal homeostasis during infectious challenge.

\section{DATA AVAILABILITY STATEMENT}

The datasets presented in this study can be found in online repositories. The names of the repository/repositories and accession number(s) can be found below: NCBI SRA BioProject, accession no: PRJNA746763.

\section{ETHICS STATEMENT}

This study was reviewed and approved by the Danish Animal Experimentation Inspectorate (License number 2015-15-020100760), and performed at the Experimental Animal Unit, University of Copenhagen according to FELASA guidelines and recommendations.

\section{AUTHOR CONTRIBUTIONS}

LM, SS, HM, PN, ST, and AW conceived and designed the experiments. LM, SS, HM, SJ, ST, and AW performed the animal study. LM performed immunological analyses. SS performed gut microbiota analyses. KS supervised qPCR analyses. LK, WK, and DN supervised microbiota gut analyses. NC performed SCFA analyses. LM, SS, and AW analyzed the data. LM and AW wrote the paper. All authors contributed to the article and approved the submitted version. 


\section{FUNDING}

This study was funded by The Danish Council for Independent Research: Technology and Production Sciences (Grant \# DFF 4184 - 00377).

\section{ACKNOWLEDGMENTS}

We thank L. Christensen (Department of Veterinary and Animal Sciences, University of Copenhagen) for her practical support throughout the study. We also thank K. Tarp (Department of

\section{REFERENCES}

1. Cortés A, Peachey L, Scotti R, Jenkins TP, Cantacessi C. Helminth-Microbiota Cross-Talk - A Journey Through the Vertebrate Digestive System. Mol Biochem Parasitol (2019) 233:111222. doi: 10.1016/j.molbiopara. 2019.111222

2. FAO and WHO. "Probiotics in Food - Health and Nutritional Properties and Guidelines for Evaluation”. In: FAO Food and Nutrition Paper 85. Rome: Food and Agriculture Organization of the United Nations and World Health Organization (2006). Available at: http://www.fao.org/3/a-a0512e.pdf.

3. Travers MA, Florent I, Kohl L, Grellier P. Probiotics for the Control of Parasites: An Overview. J Parasitol Res (2011) 2011:610769. doi: 10.1155/ 2011/610769

4. Xiao L, Estellé J, Kiilerich P, Ramayo-Caldas Y, Xia Z, Feng Q, et al. A Reference Gene Catalogue of the Pig Gut Microbiome. Nat Microbiol (2016) 1:16161. doi: 10.1038/nmicrobiol.2016.161

5. Böhmer BM, Kramer W, Roth-Maier DA. Dietary Probiotic Supplementation and Resulting Effects on Performance, Health Status, and Microbial Characteristics of Primiparous Sows. J Anim Physiol Anim Nutr (Berl.) (2006) 90:309-15. doi: 10.1111/j.1439-0396.2005.00601.x

6. Suo C, Yin Y, Wang X, Lou X, Song D, Wang X, et al. Effects of Lactobacillus Plantarum ZJ316 on Pig Growth and Pork Quality. BMC Vet Res (2012) 8:89. doi: $10.1186 / 1746-6148-8-89$

7. Sato Y, Kuroki Y, Oka K, Takahashi M, Rao S, Sukegawa S, et al. Effects of Dietary Supplementation With Enterococcus Faecium and Clostridium Butyricum, Either Alone or in Combination, on Growth and Fecal Microbiota Composition of Post-Weaning Pigs at a Commercial Farm. Front Vet Sci (2019) 6. doi: 10.3389/fvets.2019.00026

8. Wang K, Chen G, Cao G, Xu Y, Wang Y, Yang C. Effects of Clostridium Butyricum and Enterococcus Faecalis on Growth Performance, Intestinal Structure, and Inflammation in Lipopolysaccharide-Challenged Weaned Piglets. J Anim Sci (2019) 97:4140-51. doi: 10.1093/jas/skz235

9. Konstantinov SR, Smidt H, Akkermans ADL, Casini L, Trevisi P, Mazzoni M, et al. Feeding of Lactobacillus Sobrius Reduces Escherichia Coli F4 Levels in the Gut and Promotes Growth of Infected Piglets. FEMS Microbiol Ecol (2008) 66:599-607. doi: 10.1111/j.1574-6941.2008.00517.x

10. Pérez PF, Minnaard J, Rouvet M, Knabenhans C, Brassart D, De Antoni GL, et al. Inhibition of Giardia Intestinalis by Extracellular Factors From Lactobacilli: An In Vitro Study. Appl Environ Microbiol (2001) 67:5037-42. doi: 10.1128/AEM.67.11.5037-5042.2001

11. Shukla G, Bhatia R, Sharma A. Prebiotic Inulin Supplementation Modulates the Immune Response and Restores Gut Morphology in Giardia DuodenalisInfected Malnourished Mice. Parasitol Res (2016) 115:4189-98. doi: 10.1007/ s00436-016-5196-x

12. Benyacoub J, Pérez PF, Rochat F, Saudan KY, Reuteler G, Antille N, et al. Enterococcus Faecium SF68 Enhances the Immune Response to Giardia Intestinalis in Mice. J Nutr (2005) 135:1171-6. doi: 10.1093/jn/135.5.1171

13. Freeman MC, Akogun O, Belizario VJr., Brooker SJ, Gyorkos TW, Imtiaz R, et al. Challenges and Opportunities for Control and Elimination of SoilTransmitted Helminth Infection Beyond 2020. PLoS Negl Trop Dis (2019) 13: e0007201. doi: 10.1371/journal.pntd.0007201
Biotechnology and Biomedicine, Technical University of Denmark) for technical assistance with qPCR. In addition, we would like to thank Bea Nielsen (Chr. Hansen A/S) for providing all probiotic strains/mixtures used within this study, and for technical advice.

\section{SUPPLEMENTARY MATERIAL}

The Supplementary Material for this article can be found online at: https://www.frontiersin.org/articles/10.3389/fimmu.2021.793260/ full\#supplementary-material

14. Morgan ER, Aziz N-AA, Blanchard A, Charlier J, Charvet C, Claerebout E, et al. 100 Questions in Livestock Helminthology Research. Trends Parasitol (2019) 35:52-71. doi: 10.1016/j.pt.2018.10.006

15. McKay DM, Shute A, Lopes F. Helminths and Intestinal Barrier Function. Tissue Barriers (2017) 5:e1283385. doi: 10.1080/21688370.2017.1283385

16. Jensen TK, Christensen CM. Dose Related Mucosal Hyperplasia Induced by Oesophagostomum Dentatum Infection in Pigs. Can J Vet Res (1997) 61:315-8.

17. Thomas DJ, Husmann RJ, Villamar M, Winship TR, Buck RH, Zuckermann FA. Lactobacillus Rhamnosus HN001 Attenuates Allergy Development in a Pig Model. PLoS One (2011) 6:e16577. doi: 10.1371/ journal.pone.0016577

18. Petkevicius S, Bach Knudsen KE, Murrell KD, Wachmann H. The Effect of Inulin and Sugar Beet Fibre on Oesophagostomum Dentatum Infection in Pigs. Parasitology (2003) 127:61-8. doi: 10.1017/S0031182003003251

19. Petkevicius S, Murrell KD, Bach Knudsen KE, Jørgensen H, Roepstorff A, Laue A, et al. Effects of Short-Chain Fatty Acids and Lactic Acids on Survival of Oesophagostomum Dentatum in Pigs. Vet Parasitol (2004) 122:293-301. doi: 10.1016/j.vetpar.2004.03.008

20. Myhill LJ, Stolzenbach S, Hansen TVA, Skovgaard K, Stensvold CR, Andersen LO, et al. Mucosal Barrier and Th2 Immune Responses Are Enhanced by Dietary Inulin in Pigs Infected With Trichuris Suis. Front Immunol (2018) 9:2557. doi: 10.3389/fimmu.2018.02557

21. Stolzenbach S, Myhill LJ, Andersen LO, Krych L, Mejer H, Williams AR, et al. Dietary Inulin and Trichuris Suis Infection Promote Beneficial Bacteria Throughout the Porcine Gut. Front Microbiol (2020) 11:312. doi: 10.3389/ fmicb.2020.00312

22. Solano-Aguilar G, Shea-Donohue T, Madden KB, Quinoñes A, Beshah E, Lakshman S, et al. Bifidobacterium Animalis Subspecies Lactis Modulates the Local Immune Response and Glucose Uptake in the Small Intestine of Juvenile Pigs Infected With the Parasitic Nematode Ascaris Suum. Gut Microbes (2018) 9:422-36. doi: 10.1080/19490976.2018.1460014

23. Jang S, Lakshman S, Beshah E, Xie Y, Molokin A, Vinyard BT, et al. FlavanolRich Cocoa Powder Interacts With Lactobacillus Rhamnossus LGG to Alter the Antibody Response to Infection With the Parasitic Nematode Ascaris Suum. Nutrients (2017) 9(10):1113. doi: 10.3390/nu9101113

24. Andreasen A, Petersen HH, Kringel H, Iburg TM, Skovgaard K, Dawson H, et al. Immune and Inflammatory Responses in Pigs Infected With Trichuris Suis and Oesophagostomum Dentatum. Vet Parasitol (2015) 207:249-58. doi: 10.1016/j.vetpar.2014.12.005

25. Roepstorff A, Nansen P. “Chapter 3: Faecal Examinations for Parasites”. In: FAO Animal Health Manual 3: Epidemiology, Diagnosis and Control of Helminth Parasites in Swine. FAO, Rome (1998). p. 35-41.

26. Canibe N, Jensen BB. Fermented Liquid Feed and Fermented Grain to PigletsEffect on Gastrointestinal Ecology and Growth Performance. Livestock Sci (2007) 108:198-201. doi: 10.1016/j.livsci.2007.01.095

27. Slotved HC, Barnes EH, Bjørn H, Christensen CM, Eriksen L, Roepstorff A, et al. Recovery of Oesophagostomum Dentatum From Pigs by Isolation of Parasites Migrating From Large Intestinal Contents Embedded in Agar-Gel. Vet Parasitol (1996) 63:237-45. doi: 10.1016/0304-4017(95)00916-7

28. Williams AR, Krych L, Fauzan Ahmad H, Nejsum P, Skovgaard K, Nielsen DS, et al. A Polyphenol-Enriched Diet and Ascaris Suum Infection Modulate 
Mucosal Immune Responses and Gut Microbiota Composition in Pigs. PLoS One (2017) 12:e0186546. doi: 10.1371/journal.pone.0186546

29. Skovgaard K, Mortensen S, Boye M, Poulsen KT, Campbell FM, Eckersall PD, et al. Rapid and Widely Disseminated Acute Phase Protein Response After Experimental Bacterial Infection of Pigs. Vet Res (2009) 40:23. doi: 10.1051/ vetres $/ 2009006$

30. Krych L, Kot W, Bendtsen KMB, Hansen AK, Vogensen FK, Nielsen DS. Have You Tried Spermine? A Rapid and Cost-Effective Method to Eliminate Dextran Sodium Sulfate Inhibition of PCR and RT-PCR. J Microbiol Methods (2018) 144:1-7. doi: 10.1016/j.mimet.2017.10.015

31. Caporaso JG, Kuczynski J, Stombaugh J, Bittinger K, Bushman FD, Costello EK, et al. QIIME Allows Analysis of High-Throughput Community Sequencing Data. Nat Methods (2010) 7:335-6. doi: 10.1038/nmeth.f.303

32. Mao X, Gu C, Hu H, Tang J, Chen D, Yu B, et al. Dietary Lactobacillus Rhamnosus GG Supplementation Improves the Mucosal Barrier Function in the Intestine of Weaned Piglets Challenged by Porcine Rotavirus. PLoS One (2016) 11:e0146312. doi: 10.1371/journal.pone.0146312

33. Kalyana Chakravarthy S, Jayasudha R, Ranjith K, Dutta A, Pinna NK, Mande SS, et al. Alterations in the Gut Bacterial Microbiome in Fungal Keratitis Patients. PLoS One (2018) 13:e0199640. doi: 10.1371/journal.pone.0199640

34. Reda AA. Probiotics for the Control of Helminth Zoonosis. J Vet Med (2018) 2018:4178986. doi: 10.1155/2018/4178986

35. Dea-Ayuela MA, Rama-Iñiguez S, Bolás-Fernandez F. Enhanced Susceptibility to Trichuris Muris Infection of B10Br Mice Treated With the Probiotic Lactobacillus Casei. Int Immunopharmacol (2008) 8:28-35. doi: 10.1016/j.intimp.2007.10.003

36. McClemens J, Kim JJ, Wang H, Mao Y-K, Collins M, Kunze W, et al. Lactobacillus Rhamnosus Ingestion Promotes Innate Host Defense in an Enteric Parasitic Infection. Clin Vaccine Immunol (2013) 20:818. doi: 10.1128/CVI.00047-13

37. Li P, Niu Q, Wei Q, Zhang Y, Ma X, Kim SW, et al. Microbial Shifts in the Porcine Distal Gut in Response to Diets Supplemented With Enterococcus Faecalis as Alternatives to Antibiotics. Sci Rep (2017) 7:41395. doi: 10.1038/ srep41395

38. Ballou MA, Davis EM, Kasl BA. Nutraceuticals: An Alternative Strategy for the Use of Antimicrobials. Vet Clin North Am: Food Anim Pract (2019) 35:507-34. doi: 10.1016/j.cvfa.2019.08.004

39. Plaza-Diaz J, Ruiz-Ojeda FJ, Gil-Campos M, Gil A. Mechanisms of Action of Probiotics. Adv Nutr (2019) 10:S49-66. doi: 10.1093/advances/nmy063

40. Klei TR. Symposium: New Approaches in the Study of Animal Parasites. Vet Parasitol (2004) 125:147-61. doi: 10.1016/j.vetpar.2004.05.011
41. Wang B, Wu Y, Liu R, Xu H, Mei X, Shang Q, et al. Lactobacillus Rhamnosus GG Promotes M1 Polarization in Murine Bone Marrow-Derived Macrophages by Activating TLR2/MyD88/MAPK Signaling Pathway. Anim Sci J (2020) 91:e13439. doi: 10.1111/asj.13439

42. Qi SR, Cui YJ, Liu JX, Luo X, Wang HF. Lactobacillus Rhamnosus GG Components, SLP, gDNA and $\mathrm{CpG}$, Exert Protective Effects on Mouse Macrophages Upon Lipopolysaccharide Challenge. Lett Appl Microbiol (2020) 70:118-27. doi: 10.1111/lam.13255

43. Zhou D, Zhu Y-H, Zhang W, Wang M-L, Fan W-Y, Song D, et al. Oral Administration of a Select Mixture of Bacillus Probiotics Generates Tr1 Cells in Weaned F4ab/acR- Pigs Challenged With an F4+ ETEC/VTEC/EPEC Strain. Vet Res (2015) 46:95. doi: 10.1186/s13567-015-0223-y

44. Shin HS, Eom JE, Shin DU, Yeon SH, Lim SI, Lee SY. Preventive Effects of a Probiotic Mixture in an Ovalbumin-Induced Food Allergy Model. J Microbiol Biotechnol (2018) 28:65-76. doi: 10.4014/jmb.1708.08051

45. Huang C-H, Shen C-C, Liang Y-C, Jan T-R. The Probiotic Activity of Lactobacillus Murinus Against Food Allergy. J Funct Foods (2016) 25:23141. doi: 10.1016/j.jff.2016.06.006

46. Schiavi E, Barletta B, Butteroni C, Corinti S, Boirivant M, Di Felice G. Oral Therapeutic Administration of a Probiotic Mixture Suppresses Established Th2 Responses and Systemic Anaphylaxis in a Murine Model of Food Allergy. Allergy (2011) 66:499-508. doi: 10.1111/j.1398-9995.2010.02501.x

Conflict of Interest: The authors declare that the research was conducted in the absence of any commercial or financial relationships that could be construed as a potential conflict of interest.

Publisher's Note: All claims expressed in this article are solely those of the authors and do not necessarily represent those of their affiliated organizations, or those of the publisher, the editors and the reviewers. Any product that may be evaluated in this article, or claim that may be made by its manufacturer, is not guaranteed or endorsed by the publisher.

Copyright (๔) 2022 Myhill, Stolzenbach, Mejer, Krych, Jakobsen, Kot, Skovgaard, Canibe, Nejsum, Nielsen, Thamsborg and Williams. This is an open-access article distributed under the terms of the Creative Commons Attribution License (CC BY). The use, distribution or reproduction in other forums is permitted, provided the original author(s) and the copyright owner(s) are credited and that the original publication in this journal is cited, in accordance with accepted academic practice. No use, distribution or reproduction is permitted which does not comply with these terms. 